\title{
Minimum-Phase FIR Filter Design Using Real Cepstrum
}

\author{
Soo-Chang Pei, Fellow, IEEE, and Huei-Shan Lin
}

\begin{abstract}
The real cepstrum is used to design an arbitrary length minimum-phase finite-impulse response filter from a mixed-phase prototype. There is no need to start with the odd-length equiripple linear-phase filter first. Neither the phase-unwrapping nor root-finding procedure is needed. Only two fast Fourier transforms and a recursive procedure are required to find the filter's impulse response from its real cepstrum. The resulting filter's magnitude response is exactly the same as the original one even when the filter is of very high order.
\end{abstract}

Index Terms-Minimum-phase finite-impulse response (FIR) filter, phase unwrapping, real cepstrum, root finding.

\section{INTRODUCTION}

I N MANY low-delay applications of finite-impulse response (FIR) filter design such as data communication system, linear-phase characteristic is not necessary, and minimum-phase design can preserve the desired magnitude response and has the advantage of minimum delay over other counterparts with the same magnitude response.

Many methods have been developed to design minimum-phase FIR filters, especially the one proposed by Herrmann and Schuessler [1]. It starts with an odd-length linear-phase equiripple FIR filter and shifts it up by one-half the stopband's peak-to-peak ripple, which results in second-order zeros on the unit circle. The zeros inside the unit circle and each simple zero out of each pair of double unit-circle zeros are then retained to obtain the minimum-phase filter with half the degree. However, the difficulty of root-finding procedure for high-order filters limits this method, and the magnitude response becomes approximately the square root of the original one. Therefore, later research works resorted to other methods to avoid the root-finding procedure. Mian and Nainer [2] utilized the complex cepstrum to extract the minimum-phase component. In this method, only two fast Fourier transforms (FFTs) are required, but the cumbersome process of phase unwrapping is required. To avoid phase unwrapping, Pei and Lu [3] applied differential cepstrum to design the equiripple minimum-phase FIR filter, but three FFTs are required. Rather than using cepstrum, an approach based on the Newton-Raphson iterative algorithm [4] was recently recommended to find the minimum-phase

Manuscript received December 5, 2005; revised March 22, 2006. This work was supported by the National Science Council, R.O.C., under Contract NSC942213-E-002-072 and Contract NSC93-2752-E-002-006-PAE. This paper was recommended by Associate Editor Y.-P. Lin.

S.-C. Pei is with the Department of Electrical Engineering, National Taiwan University, Taipei 10617, Taiwan, R.O.C. (e-mail: pei@cc.ee.ntu.edu.tw).

H.-S. Lin is with the Graduate Institute of Communication Engineering, National Taiwan University, Taipei 10617, Taiwan, R.O.C.

Digital Object Identifier 10.1109/TCSII.2006.882193 spectral factor of polynomials. This brief emphasizes on its better accuracy than what could be obtained by root finding when there are no zeros on the unit circle. If there are, indeed, double zeros on the unit circle, however, precision will be lost.

All the aforementioned methods are aimed at designing a linear-phase equiripple FIR filter first. If this linear-phase filter is not equiripple in the stopband, we cannot merely shift up its magnitude response to get the linear-phase sequence. Moreover, the minimum-phase filter's magnitude response becomes the square root of the original one by keeping half the unit-circle zeros and all the zeros inside the unit circle. Recently, a different approach based on root moments was proposed to design minimum-phase FIR filters [5] that preserve the same magnitude response. However, it needs to start from a linear-phase FIR filter due to the complex conjugate relation between its zeros. Moreover, we need to select a proper radius of integration contour in advance to calculate moments accurately.

From the previous works of Mian and Nainer [2], we can extend it and avoid phase unwrapping by using real cepstrum. This benefits from the problem itself, that is, constructing the minimum-phase counterpart from its magnitude. It is known that a minimum-phase sequence's magnitude determines its phase [6]. Through several deductions, we will find that real cepstrum determines a sequence's minimum-phase component. Moreover, in our works, the minimum-phase filter will retain the original magnitude response exactly.

This brief is organized as follows. We first discuss several basic related concepts in Section II. Next, the formal steps for minimum-phase sequence construction using real cepstrum is summarized in Section III. We then refer to this method in an alternative viewpoint by treating it as passing the original sequence through an allpass filter in Section IV. Furthermore, several design examples are depicted to illustrate the effectiveness of this approach in Section V. In Section VI, we compare our proposed design method with several ones in the open literature [1]-[5], beside which there are still other approaches for minimum-phase filter design in recent years [7]-[10]. Finally, we make the conclusion in Section VII.

\section{BASIC CONCEPTS ON CEPSTRUM}

\section{A. Complex Cepstrum and Real Cepstrum}

Let $h(n)$ be a real sequence with $H\left(e^{j \omega}\right)$ as its Fourier transform. Its complex cepstrum $\hat{h}(n)$ and real cepstrum $\hat{c}(n)$ are defined as

$$
\begin{aligned}
\hat{H}\left(e^{j \omega}\right) & =\log \left[H\left(e^{j \omega}\right)\right] \\
& =\log \left|H\left(e^{j \omega}\right)\right|+j \arg \left[H\left(e^{j \omega}\right)\right] \\
\hat{C}\left(e^{j \omega}\right) & =\Re e\left\{\hat{H}\left(e^{j \omega}\right)\right\}=\log \left|H\left(e^{j \omega}\right)\right|
\end{aligned}
$$




$$
\begin{aligned}
& \hat{h}(n)=F^{-1}\left\{\hat{H}\left(e^{j \omega}\right)\right\} \\
& \hat{c}(n)=F^{-1}\left\{\hat{C}\left(e^{j \omega}\right)\right\}
\end{aligned}
$$

where $F^{-1}$ denotes the inverse Fourier transformation.

Note that in (1) and (3), to compute complex cepstrum, we need to perform logarithm on a complex number. The imaginary part of the complex logarithm must be continuous and without its linear-phase term to avoid ambiguity. To achieve this, we can first compute the principal value of the phase (between $-\pi$ and $\pi$ ), then unwrap the phase to a continuous one and remove the linear-phase term.

\section{B. Properties of Minimum/Maximum-Phase Sequence and Its Complex Cepstrum}

From [6], there are two useful properties.

1) If $h(n)$ is a minimum-phase sequence, its complex cepstrum $\hat{h}(n)$ will be a causal sequence. That is, $\hat{h}(n)=0$ for $n<0$.

2) If $h(n)$ is a maximum-phase sequence, its complex cepstrum $\hat{h}(n)$ will be an anticausal sequence. That is, $\hat{h}(n)=$ 0 for $n>0$.

\section{Explicit Formula Between Minimum-Phase Sequence and Its Complex Cepstrum}

An arbitrary sequence $h(n)$ and its complex cepstrum $\hat{h}(n)$ has an implicit recursive relation [6] as

$$
h(n)= \begin{cases}e^{\hat{h}(0)}, & n=0 \\ \sum_{k=-\infty}^{\infty}\left(\frac{k}{n}\right) \hat{h}(k) h(n-k), & n \neq 0 .\end{cases}
$$

Further, if $h(n)$ is a finite minimum-phase sequence, the summation stated above can be reduced to finite terms as

$$
h(n)= \begin{cases}e^{\hat{h}(0)}, & n=0 \\ \sum_{k=0}^{n}\left(\frac{k}{n}\right) \hat{h}(k) h(n-k), & n>0 .\end{cases}
$$

\section{Reconstruction of a Causal Sequence From Its Even Part}

If $h(n)$ is a causal sequence and $h_{e}(n)=[h(n)+h(-n)] / 2$ is the even part sequence of $h(n), h(n)$ can be recovered simply by $h_{e}(n)$ as

$$
h(n)=h_{e}(n) u_{+}(n), \quad \text { where } u_{+}(n)= \begin{cases}0, & n<0 \\ 1, & n=0 \\ 2, & n>0 .\end{cases}
$$

\section{E. Fourier Transform Pair Between Time- and Frequency-Domain}

Let $h_{e}(n)$ and $h_{o}(n)$ be the even part and the odd part of sequence $h(n)$, respectively. In another aspect, let $H_{R}\left(e^{j \omega}\right)$ and $H_{I}\left(e^{j \omega}\right)$ denote the real part and the imaginary part of $H\left(e^{j \omega}\right)$, which is the Fourier transform of $h(n)$.

If $h(n)$ is a real sequence, we have the following Fourier transform relations:

$$
\begin{aligned}
& h_{e}(n)=F^{-1}\left\{H_{R}\left(e^{j \omega}\right)\right\} \\
& h_{o}(n)=F^{-1}\left\{j H_{I}\left(e^{j \omega}\right)\right\}
\end{aligned}
$$

where $F^{-1}$ denotes the inverse Fourier transformation.
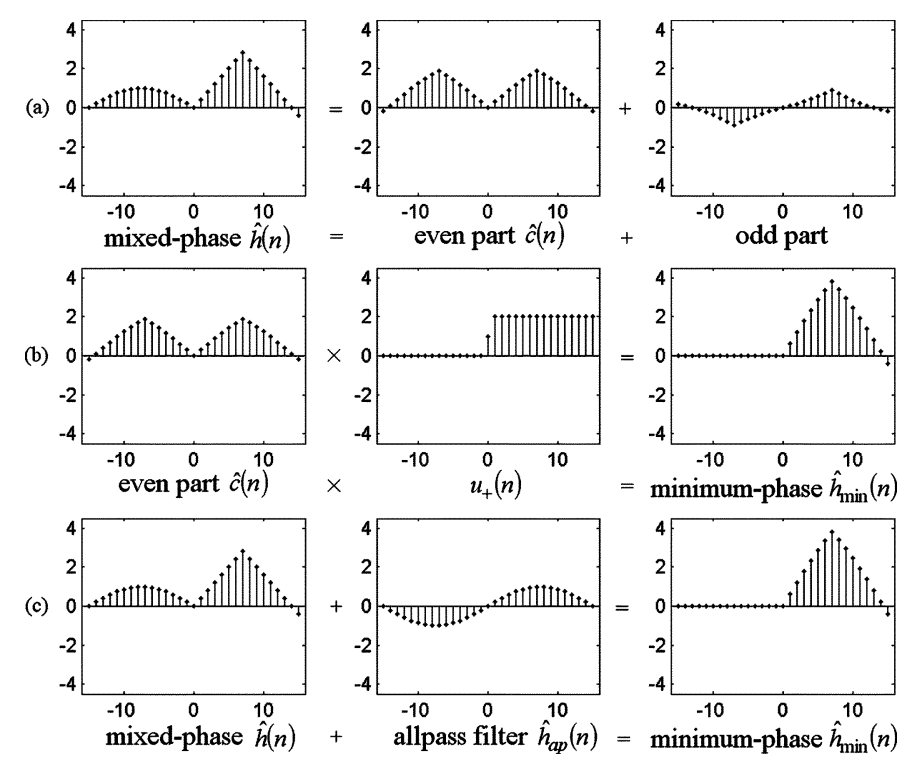

Fig. 1. Cepstrum relations among mixed-phase sequence, minimum-phase sequence, and allpass filter sequence. (a) Even part of the complex cepstrum is actually the real cepstrum. (b) Reconstruction of $\hat{h}_{\min }(n)$ from its even part $\hat{c}(n)$. (c) Reconstruction of $\hat{h}_{\min }(n)$ in allpass filtering viewpoint.

\section{F. Relation Between Complex Cepstrum and Real Cepstrum}

If $h(n)$ is a real-valued sequence, its corresponding complex cepstrum $\hat{h}(n)$ is also real valued according to the recursive relation (5). Under this constraint, we are going to relate the real cepstrum and complex cepstrum as follows: Denote $\hat{h}_{e}(n)$ as the even part of $\hat{h}(n)$, which is the complex cepstrum of $h(n)$. If we substitute $H\left(e^{j \omega}\right)$ with $\hat{H}\left(e^{j \omega}\right)=\log \left[H\left(e^{j \omega}\right)\right]$ and $H_{R}\left(e^{j \omega}\right)$ with $\hat{H}_{R}\left(e^{j \omega}\right)$ in property $E$ of Section II, we have

$$
\hat{h}_{e}(n)=F^{-1}\left\{\hat{H}_{R}\left(e^{j \omega}\right)\right\}=F^{-1}\left\{\log \left|H\left(e^{j \omega}\right)\right|\right\} .
$$

Further, using the definition of real cepstrum in (2) and (4), we have

$$
\hat{h}_{e}(n)=F^{-1}\left\{\hat{C}\left(e^{j \omega}\right)\right\}=\hat{c}(n) .
$$

That is, the real cepstrum $\hat{c}(n)$ is actually the even part of the complex cepstrum $\hat{h}(n)$, as shown in Fig. 1(a).

In this section, we only discuss the related properties of complex cepstrum and real cepstrum for our design method. There are still more literatures [11]-[17] for cepstrum analysis or algorithms for cepstrum calculation.

\section{Construction of Minimum-Phase Sequence}

The problem to be dealt with is described as follows: Given a mixed-phase real-valued sequence $h(n)$, we would like to find its minimum-phase counterpart $h_{\min }(n)$, which is a minimumphase sequence and has the same frequency magnitude response of $h(n)$. That is,

$$
\left|H_{\min }\left(e^{j \omega}\right)\right|=\left|H\left(e^{j \omega}\right)\right|
$$

where $H_{\min }\left(e^{j \omega}\right)$ and $H\left(e^{j \omega}\right)$ are the Fourier transforms of $h_{\min }(n)$ and $h(n)$, respectively. 
Definitions (1)-(4) reveal the fact that, although the respective complex cepstrum of $h_{\min }(n)$ and $h(n)$ are different according to (1) and (3), the respective real cepstrum, however, are the same according to (2), (4), and (12). In other words, since the real cepstrum is uniquely determined by the magnitude component of a sequence's frequency response, two sequences with the same magnitude response must have the same real cepstrum. Therefore

$$
\hat{c}(n)=\hat{c}_{\min }(n) .
$$

Hence, we can calculate $\hat{c}_{\min }(n)$ by performing the inverse Fourier transform on $\log \left|H\left(e^{j \omega}\right)\right|$. Further, since $\hat{h}_{\min }(n)$ is a causal sequence according to property $B$ and $\hat{c}_{\min }(n)$ is just the even part of $\hat{h}_{\min }(n)$ according to property $F$, we can apply (7) to reconstruct $\hat{h}_{\min }(n)$ from $\hat{c}_{\min }(n)$. In particular, perform

$$
\hat{h}_{\min }(n)=\hat{c}_{\min }(n) u_{+}(n)
$$

where $u_{+}(n)$ is defined in (7). This operation is illustrated in Fig. 1(b).

Finally, after $\hat{h}_{\min }(n)$ is obtained, (6) can be employed to acquire $h_{\min }(n)$. The problem solution is then found.

If $H(z)$ has its zeros on the unit circle, the region of convergence of $\log [H(z)]$ cannot include the unit circle. From this computational point of view, we should avoid the zeros existing on the unit circle. However, in practice, it is often to design digital filters with some zeros on the unit circle in the $z$-domain. We can overcome this problem by selecting a different contour $C$ slightly inside the unit circle while computing $\hat{c}(n)$ from $\hat{C}(z)$ [6]. This can be achieved equivalently by first multiplying the input $h(n)$ with an exponential sequence as

$$
h_{\alpha}(n)=\left\{\begin{array}{ll}
\alpha^{n} h(n), & n=0, \ldots, N-1 \\
0, & n=N, \ldots, L-1
\end{array} \quad \text { with } \quad L \gg 8 N\right.
$$

where $\alpha<1$ and $\alpha \cong 1$. This step will cause the radius of its zeros scaled down by the factor $\alpha$, i.e., moving its zeros slightly inside the unit circle.

Besides, even though the sequence is finite, its cepstrum sequence is still infinite [6]. Computationally, aliasing effect will occur. To reduce the aliasing effect, we must append $\alpha^{n} h(n)$ with several trailing zeros as in (15).

To design a minimum-phase filter with impulse response $h_{\min }(n)$ using the proposed method, we must start with a prototypical impulse response $h(n)$ with the same magnitude response. If the prototype is unknown, we can use the FIR filter design algorithm such as the Parks-McClellan program [18] to design the linear-phase equiripple FIR filter prototype $h(n)$ according to the specifications, including the filter's length, maximum ripples on the passband and the stopband, and the cutoff frequencies.

Now, we summarize the overall steps for constructing the minimum-phase sequence $h_{\min }(n)$ from any mixed-phase or linear-phase sequence $h(n)$ as follows.

1) Choose $\alpha \leq 1$ and $\alpha \cong 1$ to move the zeros slightly inside on the unit circle.

2) Perform $L$-point $(\mathrm{FFT})_{L}$ on $h_{\alpha}(n)=\alpha^{n} h(n)$, $n=0,1, \ldots,(N-1)$, to get $H_{\alpha}(k), k=0,1, \ldots,(L-1)$, $L \gg 8 N$.
3) Perform (IFFT) $)_{L}$ on $\log \left|H_{\alpha}(k)\right|$ to get $\hat{c}_{\alpha}(n)$, which is equal to $\hat{c}_{\alpha, \min }(n)$.

4) Construct $\hat{h}_{\alpha, \min }(n)$ from $\hat{c}_{\alpha, \min }(n)$ using (12).

5) Compute $h_{\alpha, \min }(n)$ from $\hat{h}_{\alpha, \min }(n)$ using (6).

6) Rescale $h_{\alpha, \min }(n)$ to get $h_{\min }(n)=h_{\alpha, \min }(n) \alpha^{-n}$.

\section{AllPASS FILTERING VIEWPOINT}

In this section, we provide another viewpoint on the work presented above. In fact, the proposed process in the previous section is equivalent to passing the mixed-phase or linear-phase sequence through a proper allpass filter to acquire a minimumphase output sequence. Intuitively, this can be inferred from the fact that the mixed-phase or the linear-phase sequence and its minimum-phase counterpart have the same magnitude response with different phase response. Formally, we can prove this by considering the characteristics of an allpass filter's complex cepstrum.

An allpass filter's transfer function $H_{\mathrm{ap}}(z)$ can be expressed as

$$
H_{\mathrm{ap}}(z)=\frac{\prod_{k=1}^{N}\left(1-a_{k} z^{-1}\right)}{\prod_{k=1}^{N}\left(1-a_{k} z\right)} z^{N}, \quad \text { where } \quad\left|a_{k}\right|<1 .
$$

We can drop the linear-phase term $z^{N}$ to compute its complex cepstrum as follows:

$$
\begin{aligned}
\log \left[H_{\mathrm{ap}}(z)\right]= & -\sum_{k=1}^{N} \log \left(1-a_{k} z\right)+\sum_{k=1}^{N} \log \left(1-a_{k} z^{-1}\right) \\
= & \sum_{k=1}^{N} \sum_{n=1}^{\infty} \frac{a_{k}^{n}}{n} z^{n}-\sum_{k=1}^{N} \sum_{n=1}^{\infty} \frac{a_{k}^{n}}{n} z^{-n} \\
= & \sum_{n=-\infty}^{-1}\left(\sum_{k=1}^{N} \frac{-a_{k}^{-n}}{n}\right) z^{-n} \\
& +\sum_{n=1}^{\infty}\left(\sum_{k=1}^{N} \frac{-a_{k}^{n}}{n}\right) z^{-n} .
\end{aligned}
$$

Thus

$$
\hat{h}_{\mathrm{ap}}(n)=\left\{\begin{array}{cc}
\sum_{k=1}^{N} \frac{-a_{k}^{n}}{n}, & n>0 \\
0, & n=0 \\
\sum_{k=1}^{N} \frac{-a_{k}^{-n}}{n}, & n<0 .
\end{array}\right.
$$

Notice that an allpass filter's complex cepstrum is an odd sequence. From the previous discussion, while we drop the noncausal part of the mixed-phase sequence's complex cepstrum, it can be viewed as follows: To apply an allpass filter whose noncausal part of complex cepstrum is just the negative noncausal part of the mixed-phase sequence's complex cepstrum as shown in Fig. 1(c). Therefore, the two noncausal parts of complex cepstrum will be added and canceled to be zero. Moreover, if we consider the $H(z)$ 's zero location, we find that, by multiplying $H(z)$ with $H_{\mathrm{ap}}(z)$, the effect is that $H(z)$ 's zeros lying outside the unit circle will be canceled by the poles of $H_{\mathrm{ap}}(z)$ and be reflected inside the unit circle at their reciprocal conjugate locations.

\section{DESIGN EXAMPLES}

In the following, three examples are given to illustrate the design of minimum-phase FIR filter by the proposed method. 

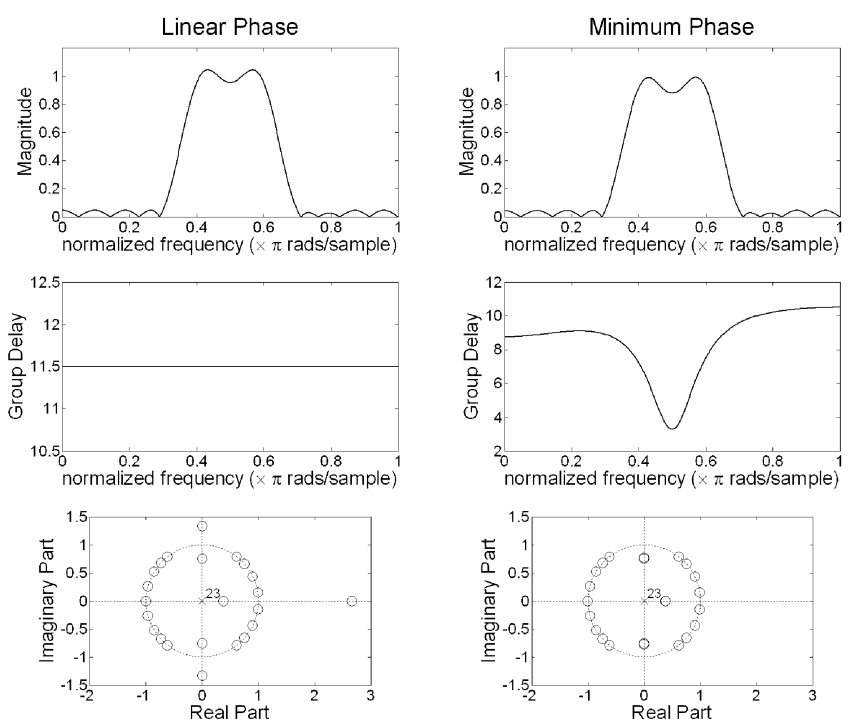

Fig. 2. Equiripple bandpass linear-phase and minimum-phase filters with length 24. (a) Amplitude response. (b) Group-delay response. (c) Zero-pole plot.
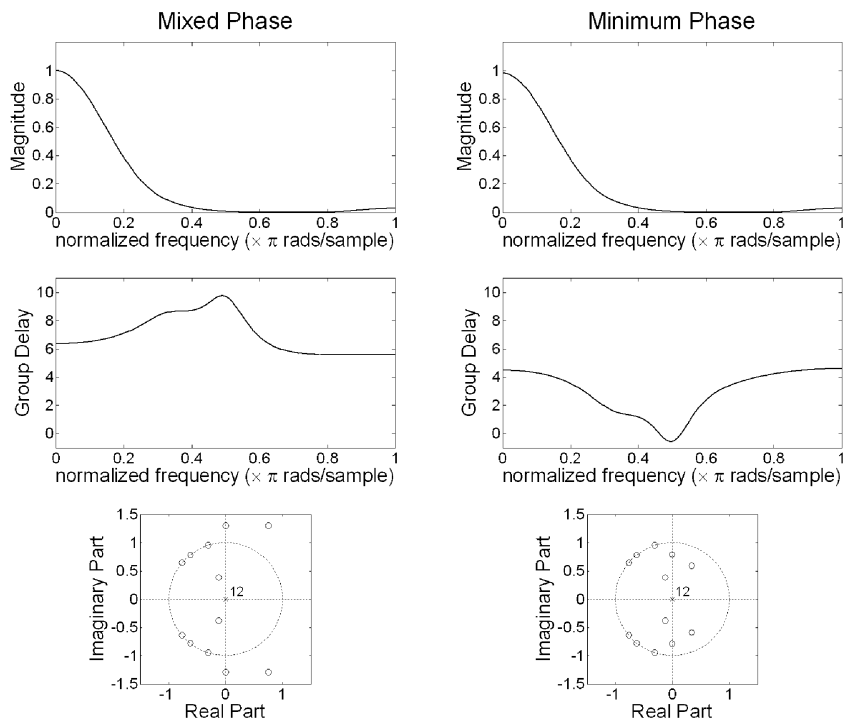

Fig. 3. Low-pass mixed-phase and minimum-phase filters with length 13. (a) Amplitude response. (b) Group-delay response. (c) Zero-pole plot.

Among the three examples, the first one is the linear-phase equiripple even-length bandpass filter with length 24 . The second one is a low-pass mixed-phase odd-length filter with length 13 , which emphasizes that we need not start with a linear-phase filter to accomplish our work. The third example is a high-order low-pass filter with length 128. In the three cases, their frequency magnitude/group-delay response and zero-pole plot before and after the minimum-phase design procedure are shown in Figs. 2-4, respectively. In the following, we discuss several points revealed from these design examples.

First, the magnitude response of each minimum-phase sequence, as desired, remains the same as the corresponding prototype sequence. This results from the fact that, in the design process, we have employed the real cepstrum of the prototype sequence, which possesses equivalent information to their frequency magnitude response, as the real cepstrum of desired minimum-phase sequence. Second, from each plot of group-delay
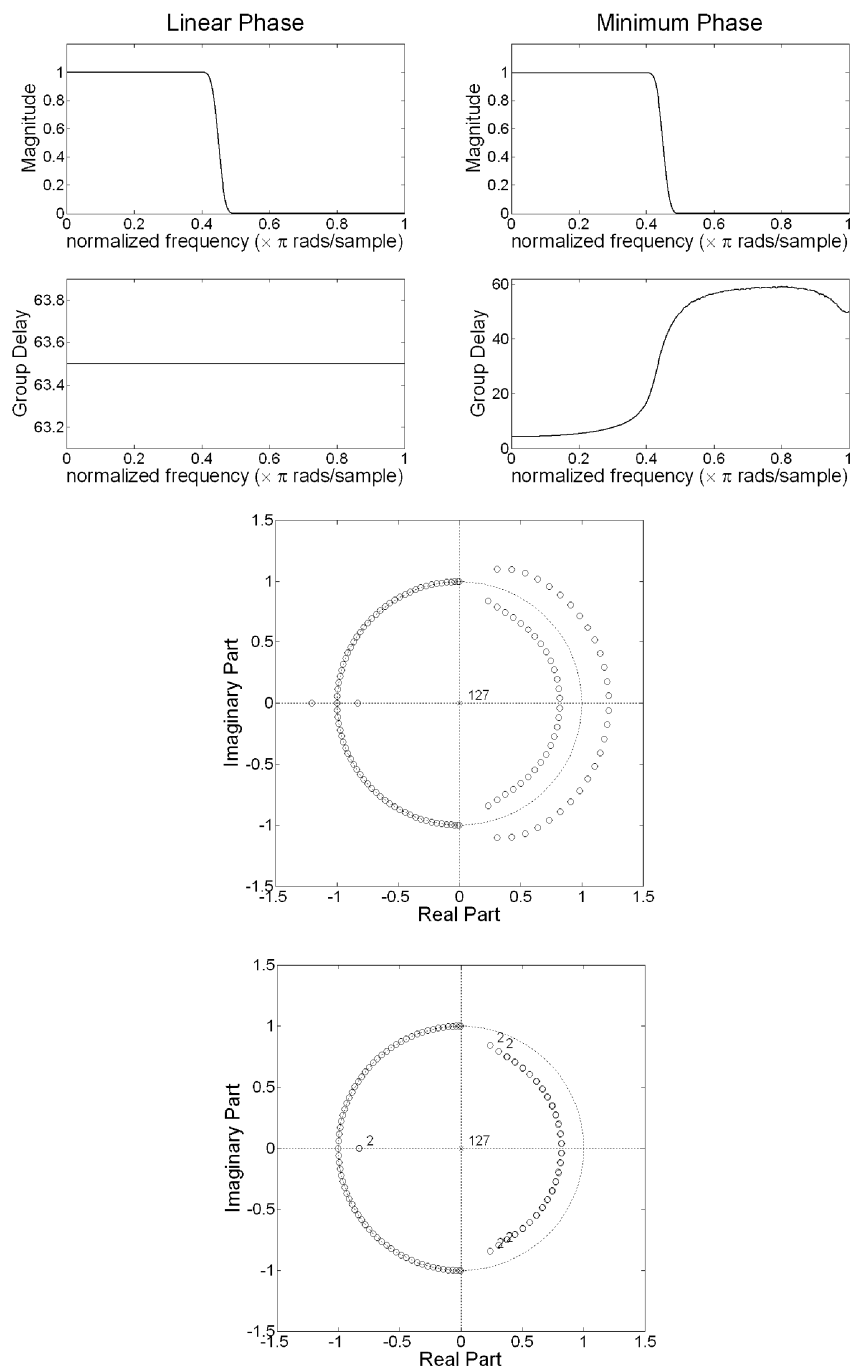

Fig. 4. Equiripple low-pass linear-phase and minimum-phase high-order filters with length 128. (a) Amplitude response. (b) Group-delay response. (c) Zeropole plot.

response, it is obvious that the group delay of the minimumphase sequence is indeed less than that of one of the corresponding prototype sequences. This accords with the fact that a minimum-phase sequence is also a minimum-group-delay sequence [6]. Third, the zeros of the resultant sequences' $z$-transform, except for the ones on the unit circle, are all inside the unit circle, which proves that the resultant sequences are minimum-phase sequences. In the design process, the zeros outside the unit circle are reflected into their conjugate reciprocal locations. In the case of linear-phase prototypes, as shown in Figs. 2 and 4, the $z$-transform of resultant minimum-phase sequences will have double zeros inside the unit circle. In the case of mixed-phase prototype, as shown in Fig. 3, it is more obvious that the zeros outside the unit circle are reflected into their conjugate reciprocal locations. Besides, in all the examples, the unit-circle zeros remain unchanged after the design process. Lastly, in the case of high-order sequence as shown in Fig. 4, this design process still performs well. At the same time, it saves much effort since we need not find the roots of its $z$-transform. 
TABLE I

Comparison Between the Proposed Method and SEVERAL EXISTING METHODS IN THE OPEN LITERATURE

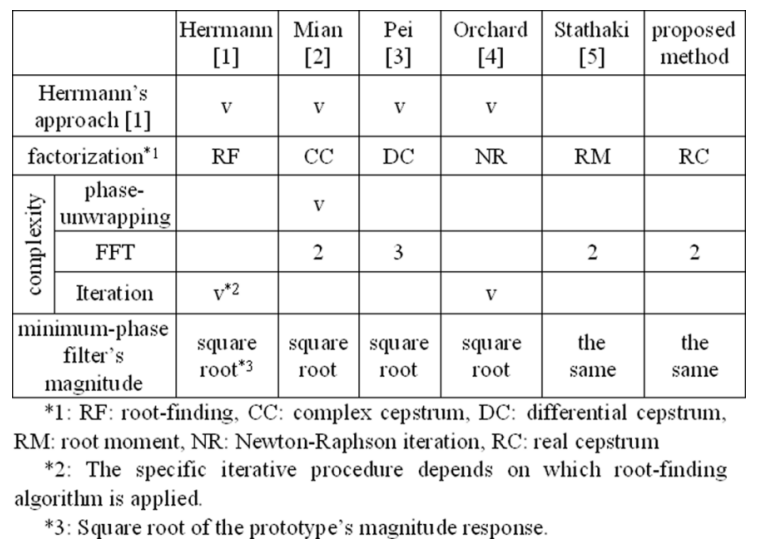

\section{Performance ANd COMPLEXITy COMPARISON}

In Table I, we compare the new method with other methods proposed in the open literature [1]-[5]. Note that the methods [2]-[4] are all mainly based on Herrmann's design procedure [1], in which the frequency response of odd-length equiripple linear-phase prototype filter are shifted up by one-half of its stopband's peak-to-peak ripple to acquire the second-order zeros on the unit circle of the $z$-plane. Then, the zeros belonging to the minimum-phase component is retained by the specific factorization approach. Therefore, the resultant magnitude response will become approximately the square-root magnitude of the original prototype. In our design procedure and the procedure in [5], the efforts required are merely two FFTs and a recursive procedure to compute the impulse response either from real cepstrum or from root moments. Therefore, the complexities are the lowest. However, there is a conspicuous difference between [5] and our proposed method; unlike [5], in which a linear-phase prototype is required, our design procedure can begin with a general mixed-phase prototype.

\section{CONCLUSION}

We have introduced a simple effective method to construct a minimum-phase FIR filter from a mixed-phase filter. During the process, there's no need to unwrap the phase or find the roots. Neither do we need to begin with an odd-length equiripple linear-phase prototype filter and get the square-root magnitude response. Note that coefficient scaling is used to handle the unit-circle zeros' numerical problem. Furthermore, while we compute the real cepstrum sequence, zero padding is necessary to reduce the aliasing effect. The resultant minimum-phase filter magnitude response will be exactly the same as the original one even when the filter is of high order.

\section{REFERENCES}

[1] O. Herrmann and W. Schuessler, "Design of nonrecursive digital filters with minimum phase," Electron. Lett., vol. 6, no. 11, pp. 329-330, May 1970.

[2] G. A. Mian and A. P. Nainer, "A fast procedure to design equiripple minimum-phase FIR filters," IEEE Trans. Circuits Syst., vol. CAS-29, no. 5, pp. 327-331, May 1982.

[3] S.-C. Pei and S.-T. Lu, "Design of minimum-phase FIR filters by differential cepstrum," IEEE Trans. Circuits Syst., vol. CAS-33, no. 5, pp. 570-576, May 1986.

[4] H. J. Orchard and A. N. Willson, "On the computation of a minimumphase spectral factor," IEEE Trans. Circuits Syst. I, Fundam. Theory Appl., vol. 50, no. 3, pp. 365-375, Mar. 2003

[5] T. Stathaki and I. Fotinopoulos, "Equiripple minimum phase FIR filter design from linear phase systems using root moments," IEEE Trans. Circuits Syst. II, Analog Digit. Signal Process., vol. 48, no. 6, pp. 580-587, Jun. 2001.

[6] A. V. Oppenheim and R. W. Schafer, Discrete-Time Signal Processing. Englewood Cliffs, NJ: Prentice-Hall, 1975, pp. 501-511.

[7] N. Damera-Venkata, B. L. Evans, and S. R. McCaslin, "Design of optimal minimum-phase digital FIR filters using discrete Hilbert transforms," IEEE Trans. Signal Process., vol. 48, no. 5, pp. 1491-1495, May 2000.

[8] H. H. Dam, S. Nordebo, and L. Svensson, "Design of minimum-phase digital filters as the sum of two allpass functions using the cepstrum technique," IEEE Trans. Signal Process., vol. 51, no. 3, pp. 726-731, Mar. 2003.

[9] M. Okuda, I. R. Khan, M. Ikehara, and S. Takahashi, "Quasi-equiripple approximation of minimum phase FIR filters by updating desired response," Proc. Inst. Electr. Eng. Vis. Image Signal Process., vol. 151, no. 3, pp. 164-169, Jun. 2004.

[10] T. Nagarajan, K. Prasad, and H. A. Murthy, "Minimum phase signal derived from root cepstrum," Electron. Lett., vol. 39, no. 12, pp. 941-942, Jun. 2003.

[11] F. H. Pienaar, "New cepstrum algorithm," in Proc. IEEE South African Symp. Commun. and Signal Process., Oct. 1994, pp. 105-107.

[12] E. Krajnik, "On time-domain deconvolution and the computation of the cepstrum," in Proc. IEEE Int. Symp. Circuits and Syst., 1995, vol. 3, pp. 1908-1911.

[13] T. K. Bysted, "Aliasing in the complex cepstrum of linear-phase signals," in Proc. Int. Conf. Inf., Commun. and Signal Process., Sep. 1997, vol. 3, pp. 1598-1602.

[14] D. Zazula, "New definition of cepstral calculus," in Proc. 4th Int. Conf. Signal Process., 1998, vol. 1, pp. 27-30.

[15] M. C. Steckner and D. J. Drost, "Fast cepstrum analysis using the Hartley transform," IEEE Trans. Acoust., Speech, Signal Process., vol. 37, no. 8, pp. 1300-1302, Aug. 1989.

[16] R. T. Sokolov and J. C. Rogers, "Time-domain cepstral transformations,” IEEE Trans. Signal Process., vol. 41, no. 3, pp. 1161-1169, Mar. 1993.

[17] J. Bednar and T. Watt, "Calculating the complex cepstrum without phase unwrapping or integration," IEEE Trans. Acoust., Speech, Signal Process., vol. ASSP-33, no. 4, pp. 1014-1017, Aug. 1985.

[18] T. W. Parks and J. H. McClellan, "A program for the design of linear phase finite impulse response filters," IEEE Trans. Audio Electroacoust., vol. AU-20, no. 3, pp. 195-199, Aug. 1972. 